\title{
Impulsive Stabilization of Dynamic Equations on Time Scales
}

\author{
Yinhua Cui, ${ }^{1}$ Sung Kyu Choi, ${ }^{2}$ and Namjip Koo ${ }^{2}$ \\ ${ }^{1}$ Institute of Business and Economic Research, Harbin University of Commerce, Harbin 150076, China \\ ${ }^{2}$ Department of Mathematics, Chungnam National University, Daejeon 305-764, Republic of Korea
}

Correspondence should be addressed to Namjip Koo; njkoo@cnu.ac.kr

Received 24 February 2014; Accepted 30 July 2014; Published 14 October 2014

Academic Editor: Paul W. Eloe

Copyright (C) 2014 Yinhua Cui et al. This is an open access article distributed under the Creative Commons Attribution License, which permits unrestricted use, distribution, and reproduction in any medium, provided the original work is properly cited.

In this paper we study the impulsive stabilization of dynamic equations on time scales via the Lyapunov's direct method. Our results show that dynamic equations on time scales may be $\psi$-exponentially stabilized by impulses. Furthermore, we give some examples to illustrate our results.

\section{Introduction}

Differential equations with impulse effect provide an adequate mathematical description of various real-world phenomena in physics, engineering, biology, economics, neutral network, social sciences, and so forth. Since the 1960s, the theory of impulsive differential or difference equations has been studied by many authors [1-3].

Aulbach and Hilger $[4,5]$ introduced the theory of time scales (measure chains) in order to create a theory that can unify continuous and discrete analysis. The theory of dynamic systems on time scales has been developed as a generalization of both continuous and discrete dynamic systems simultaneously and applied to many different fields of mathematics $[6,7]$.

It is widely known that the various types of stability of nonlinear impulsive differential equations or impulsive difference equations can be characterized by using Lyapunov's second method and inequalities [8-11]. In recent years, some authors studied the stability of impulsive dynamic systems on time scales [12-16]. Furthermore, Hatipoğlu et al. [12] studied the $\psi$-exponential stability of nonlinear impulsive dynamic equations on time scales. Liu [17] investigated the impulsive stabilization of nonlinear systems by employing Lyapunov's direct method and obtained sufficient conditions for both stabilization and destabilization.

In this paper we study the impulsive stabilization of dynamic equations on time scales via the Lyapunov's direct method. Our results show that dynamic equations on time scales may be $\psi$-exponentially stabilized by impulses. We give some examples to illustrate our results.

\section{Preliminaries}

We refer the reader to [6] for all the basic definitions and results from time scales calculus that we will use in the sequel (e.g., delta differentiability, rd-continuity, and exponential function and its properties).

It is assumed throughout that a time scale $\mathbb{\mathbb { }}$ will be unbounded above and $\mu(t)$ is bounded. Let $\mathbb{R}^{n}$ be the $n$ dimensional real Euclidean space. $C_{\text {rd }}\left(\mathbb{T} \times \mathbb{R}^{n}, \mathbb{R}^{n}\right)$ denotes the set of all rd-continuous functions from $\mathbb{T} \times \mathbb{R}^{n}$ to $\mathbb{R}^{n}$ and $\mathbb{R}_{+}=[0, \infty)$. Also, for any $t_{0} \in \mathbb{T}$, let $\mathbb{T}_{t_{0}}:=\left[t_{0}, \infty\right) \cap \mathbb{T}$. We denote by $\mathscr{R}$ (resp., $\mathscr{R}^{+}$) the set of all regressive (resp., positively regressive) functions from $\mathbb{T}$ to $\mathbb{R}$. The set of all rdcontinuous and regressive functions from $\mathbb{T}$ to $\mathbb{R}$ is denoted by $C_{\text {rd }} \mathscr{R}(\mathbb{T}, \mathbb{R})$. Also, let

$$
\begin{aligned}
& C_{\mathrm{rd}} \mathscr{R}^{+}(\mathbb{T}, \mathbb{R}) \\
& \quad:=\left\{p \in C_{\mathrm{rd}} \mathscr{R}(\mathbb{T}, \mathbb{R}): 1+\mu(t) p(t)>0 \forall t \in \mathbb{T}\right\} .
\end{aligned}
$$

We consider the impulsive dynamic system with impulses at constant times

$$
\begin{gathered}
x^{\Delta}(t)=f(t, x), \quad t \neq t_{k}, t \in \mathbb{T}_{t_{0}} ; \\
\Delta x(t):=x\left(t^{+}\right)-x(t)=I_{k}(x(t)), \quad t=t_{k} ; \\
x\left(t_{0}\right)=x_{0},
\end{gathered}
$$


with the following conditions:

(i) $t_{0}<t_{1}<t_{2}<\cdots<t_{k}<\cdots$, with $\lim _{k \rightarrow \infty} t_{k}=$ $\infty, t_{k} \in \mathbb{T}$ for $k \in \mathbb{N}$.

(ii) The function $f: \mathbb{T} \times \mathbb{R}^{n} \rightarrow \mathbb{R}^{n}$ is rd-continuous in $\left(t_{k-1}, t_{k}\right] \times \mathbb{R}^{n}$ and $f(t, 0)=0$ for $t \in \mathbb{T}$.

(iii) The function $I_{k}: \mathbb{R}^{n} \rightarrow \mathbb{R}^{n}$ is continuous and $I_{k}(0)=$ 0 for $k \in \mathbb{N}$;

(iv) $x\left(t_{k}^{+}\right)$represents the right limit of $x(t)$ at $t=t_{k}$.

The solution of the impulsive dynamic equation with impulse effect (2) depends not only on the initial condition $\left(t_{0}, x_{0}\right)$ but also on the moments of impulses $t_{k}$ for each $k \in \mathbb{N}$. Let $x(t)=x\left(t, t_{0}, x_{0}\right)$ be the unique solution of (2) satisfying the initial condition $x\left(t_{0}, t_{0}, x_{0}\right)=x_{0}$. For the existence and continuation of solutions of impulsive dynamic equations, see $[3,18]$.

We need the following well-known impulsive inequality of Gronwall's type to prove our main results.

Lemma 1 (see [14]). Let $t_{0} \in \mathbb{T}$, let $u \in C_{\text {rd }} \mathscr{R}\left(\mathbb{T}_{t_{0}}, \mathbb{R}_{+}\right)$, let $p \in C_{\text {rd }} \mathscr{R}^{+}\left(\mathbb{T}_{t_{0}}, \mathbb{R}\right)$, and let $c, b_{k} \in \mathbb{R}_{+}$for each $k \in \mathbb{N}$. Then,

$$
u(t) \leq c+\int_{t_{0}}^{t} p(s) u(s) \Delta s+\sum_{t_{0}<t_{k}<t} b_{k} u\left(t_{k}\right), \quad t \in \mathbb{T}_{t_{0}}
$$

implies

$$
u(t) \leq c \prod_{t_{0}<t_{k}<t}\left(1+b_{k}\right) e_{p}\left(t, t_{0}\right), \quad t \in \mathbb{T}_{t_{0}} .
$$

Lemma 2 (see [19]). For every positive constant $\lambda$ with $-\lambda \epsilon$ $\mathscr{R}^{+}$, the following inequalities hold:

$$
e_{-\lambda}\left(t, t_{0}\right) \leq e^{-\lambda\left(t-t_{0}\right)} \leq e_{\ominus \lambda}\left(t, t_{0}\right), \quad t \in \mathbb{T}_{t_{0}},
$$

where $\ominus \lambda=-\lambda /(1+\mu(t) \lambda)$.

Lemma 3 (see [6]). If $p, q \in \mathscr{R}$, then we have, for all $t, s, r \in \mathbb{T}$,

(i) $e_{0}(t, s)=1$ and $e_{p}(t, t)=1$;

(ii) $e_{p}(\sigma(t), s)=(1+\mu(t) p(t)) e_{p}(t, s)$;

(iii) $1 / e_{p}(t, s)=e_{\ominus p}(t, s)$ and $e_{p}(t, s)=1 / e_{p}(s, t)=$ $e_{\ominus p}(s, t)$;

(iv) $e_{p}(t, s) e_{p}(s, r)=e_{p}(t, r)$;

(v) $e_{p}(t, s) e_{q}(t, s)=e_{p \oplus q}(t, s)$ and $e_{p}(t, s) / e_{q}(t, s)=$ $e_{p \ominus q}(t, s)$.

Akinyele [20] introduced the notion of $\psi$-stability of degree $k$ with respect to a function $\psi \in C\left(\mathbb{R}_{+}, \mathbb{R}_{+}\right)$, increasing and differentiable on $\mathbb{R}_{+}$and such that $\psi(t) \geq 1$ for $t \geq 0$ and $\lim _{t \rightarrow \infty} \psi(t)=b, b \in[1, \infty)$.

Now, we give notions of $\psi$-exponential, $\psi$-uniformly exponential, and $\psi$-globally exponential stability for solutions of nonlinear impulsive dynamic equations on time scales.
Definition 4 (see [12]). Let $\psi \in C_{\mathrm{rd}}\left(\mathbb{T}, \mathbb{R}_{+}\right)$. System (2) is called $\psi$-exponentially stable if any solution $x\left(t, t_{0}, x_{0}\right)$ of $(2)$ satisfies

$$
\left\|\psi(t) x\left(t, t_{0}, x_{0}\right)\right\| \leq \beta\left(\left\|x_{0}\right\|, t_{0}\right)\left[e_{-\lambda}\left(t, t_{0}\right)\right]^{d}, \quad t \in \mathbb{T}_{t_{0}},
$$

where the function $\beta(h, t): \mathbb{R}_{+} \times \mathbb{T} \rightarrow \mathbb{R}_{+}$is increasing in $h \in \mathbb{R}_{+}, \lambda>0,-\lambda \in \mathscr{R}^{+}$, and $d$ is a positive constant.

Moreover, system (2) is said to be $\psi$-uniformly exponentially stable if $\beta$ is independent of $t_{0}$.

System (2) is said to be $\psi$-globally exponentially stable if system (2) is $\psi$-exponentially stable for each $\left(t_{0}, x_{0}\right) \in \mathbb{T}_{t_{0}} \times$ $\mathbb{R}^{n}$ and the function $\beta$ is independent on each $t_{0}$ and $x_{0}$ in the definition of $\psi$-exponential stability; that is, there exist constants $\lambda>0$ with $-\lambda \in \mathscr{R}^{+}$and $M \geq 1$ such that for any initial value $\left(t_{0}, x_{0}\right) \in \mathbb{T}_{0} \times \mathbb{R}^{n}$,

$$
\left\|\psi(t) x\left(t, t_{0}, x_{0}\right)\right\| \leq M\left\|\psi\left(t_{0}\right) x_{0}\right\|\left[e_{-\lambda}\left(t, t_{0}\right)\right]^{d}, \quad t \in \mathbb{T}_{t_{0}},
$$

where $x\left(t, t_{0}, x_{0}\right)$ is any solution of system (2).

Remark 5. System (2) is exponentially stable if we set $\psi(t)=1$ in the definition of $\psi$-exponential stability.

Moreover, system (2) is uniformly exponentially stable if we set $\psi(t)=1$ in the definition of $\psi$-uniformly exponential stability.

For the Lyapunov-like function $V \in C_{\text {rd }}\left(\mathbb{\nabla} \times \mathbb{R}^{n}, \mathbb{R}_{+}\right)$, we recall the following definition.

Definition 6 (see [7, Definition 3.1.1]). We define the generalized derivative $D^{+} V_{(2)}^{\Delta}(t, x(t))$ of $V(t, x)$ relative to system (2) as follows: given $\varepsilon>0$, there exists a neighborhood $U$ of $t \in \mathbb{T}$ such that

$$
\begin{aligned}
& \frac{1}{\sigma(t)-s}[V(\sigma(t), x(\sigma(t))) \\
& \quad-V(s, x(\sigma(t))-(\sigma(t)-s) f(t, x(t)))] \\
& <D^{+} V_{(2)}^{\Delta}(t, x(t))+\varepsilon, \quad s \in U, s>t
\end{aligned}
$$

where $x(t)$ is any solution of system (2) and the upper right Dini derivative $V_{*}^{\Delta}(t)$ of $V_{*}(t)$ is given by

$$
V_{*}^{\Delta}(t)= \begin{cases}\varlimsup_{\eta \rightarrow 0^{+}, \eta+t \in \mathbb{T}} \frac{V_{*}(t+\eta)-V_{*}(t)}{\eta}, & \text { if } t=\sigma(t), \\ \frac{V_{*}(\sigma(t))-V_{*}(t)}{\mu(t)}, & \text { if } t<\sigma(t),\end{cases}
$$

where $V_{*}(t)=V(t, x(t))$.

Then it is well-known that

$$
D^{+} V_{(2)}^{\Delta}(t, x(t))=V_{*}^{\Delta}(t)
$$

if $V(t, x)$ is Lipschitzian in $x$ for each $t \in \mathbb{T}[21]$. 
In case $t \in \mathbb{T}$ is right-dense, we have

$$
\begin{aligned}
D^{+} & V_{(2)}^{\Delta}(t, x(t)) \\
& =D^{+} V_{(2)}(t, x(t)) \\
& =\varlimsup_{\eta \rightarrow 0+\eta} \frac{1}{\eta}[V(t+\eta, x(t)+\eta f(t, x(t)))-V(t, x(t))] \\
& =\varlimsup_{\eta \rightarrow 0^{+}, \eta+t \in \mathbb{T}} \frac{V(t+\eta, x(t+\eta))-V(t, x(t))}{\eta} \\
& =D^{+} V_{*}(t) .
\end{aligned}
$$

In case $t \in \mathbb{T}$ is right-scattered and $V(t, x(t))$ is continuous at $t$, we have

$$
D^{+} V_{(2)}^{\Delta}(t, x(t))=\frac{1}{\mu(t)}[V(\sigma(t), x(\sigma(t)))-V(t, x(t))] .
$$

In fact, if $x(t)$ is a solution of system (2), then we have

$$
\begin{aligned}
V^{\Delta}(t, x(t)) \\
=V^{\Delta_{t}}(t, x(t)) \\
\quad+\left[\int_{0}^{1} D_{2} V\left(\sigma(t), x(t)+\eta \mu(t) x^{\Delta}(t)\right) \mathrm{d} \eta\right] x^{\Delta}(t) \\
=V^{\Delta_{t}}(t, x(\sigma(t))) \\
\quad+\left[\int_{0}^{1} D_{2} V\left(t, x(t)+\eta \mu(t) x^{\Delta}(t)\right) \mathrm{d} \eta\right] x^{\Delta}(t)
\end{aligned}
$$

by the chain rule of a differentiable function $V(t, x(t))[22$, Theorem 1].

Definition 7 (see [23]). $V: \mathbb{T} \times \mathbb{R}^{n} \rightarrow \mathbb{R}_{+}$is said to belong to the class $v_{0}$ if

(i) $V$ is rd-continuous in $\left(\left(t_{k-1}, t_{k}\right] \cap \mathbb{T}\right) \times \mathbb{R}^{n}$ and for each $x \in \mathbb{R}^{n}, t \in\left(t_{k-1}, t_{k}\right] \cap \mathbb{T}, k \in$ $\mathbb{N}, \lim _{(t, y) \rightarrow\left(t_{k}^{+}, x\right)} V(t, y)=V\left(t_{k}^{+}, x\right)$ exists.

(ii) $V(t, x)$ is locally Lipschizian in $x \in \mathbb{R}^{n}$ and $V(t, 0)=0$ for $t \in \mathbb{T}$.

\section{Main Results}

In this section we investigate $\psi$-exponential stability for impulsive dynamic equations on time scales via Lyapunov's direct method.

The following result shows that dynamic equations on time scales may be $\psi$-exponentially stabilized by impulses. It is adapted from Theorem 3.1 in [11].

Theorem 8. Assume that there exists a function $V \in \nu_{0}$ and constants $p, q, c, c_{1}, c_{2}>0$ and $\alpha>0, \lambda>c$ with $-\lambda \in \mathscr{R}^{+}$ such that the following conditions hold: (i) $c_{1}\|\psi(t) x\|^{p} \leq V(t, x) \leq c_{2}\|\psi(t) x\|^{q}$ for $(t, x) \in \mathbb{T}_{t_{0}} \times \mathbb{R}^{n}$;

(ii) $V^{\Delta}(t, x) \leq c V(t, x)$ for all $t \in\left(t_{k-1}, t_{k}\right] \cap \mathbb{T}_{t_{0}}$;

(iii) $V\left(t_{k}^{+}, x\left(t_{k}\right)+I_{k}\left(x\left(t_{k}\right)\right)\right) \leq d_{k} V\left(t_{k}, x\left(t_{k}\right)\right)$, where each $d_{k}$ is a positive constant;

(iv) $0<t_{k}-t_{k-1}<\alpha$ and $d_{k}<e_{-\lambda}\left(t_{k+1}, t_{k}\right) e^{-\alpha \lambda}$ for each $k \in \mathbb{N}$.

Then the zero solution of system (2) is $\psi$-exponentially stable.

Proof. Let $x(t)=x\left(t, t_{0}, x_{0}\right)$ be any solution of system (2) with initial value $x\left(t_{0}\right)=x_{0}$, and $V_{*}(t)=V(t, x(t))$.

We will show that

$$
\begin{aligned}
& V_{*}(t) \\
& \quad \leq M_{1}\left\|\psi\left(t_{0}\right) x_{0}\right\|^{q} e_{-\lambda}\left(t_{k}, t_{0}\right), \quad t \in\left(t_{k-1}, t_{k}\right] \cap \mathbb{T}_{t_{0}}, k \in \mathbb{N} .
\end{aligned}
$$

We can choose $M_{1} \geq 1$ such that

$$
\begin{aligned}
c_{2}\left\|\psi\left(t_{0}\right) x_{0}\right\|^{q} & <M_{1}\left\|\psi\left(t_{0}\right) x_{0}\right\|^{q} e_{-\lambda}\left(t_{1}, t_{0}\right) e^{-\alpha c} \\
& <M_{1}\left\|\psi\left(t_{0}\right) x_{0}\right\|^{q} e_{-\lambda}\left(t_{1}, t_{0}\right) .
\end{aligned}
$$

We first show that

$$
V_{*}(t) \leq M_{1}\left\|\psi\left(t_{0}\right) x_{0}\right\|^{q} e_{-\lambda}\left(t_{1}, t_{0}\right), \quad t \in\left[t_{0}, t_{1}\right] \cap \mathbb{T}_{t_{0}} .
$$

In view of conditions (i) and (15), we have

$$
\begin{aligned}
V_{*}(t) & \leq V_{*}\left(t_{0}\right) e_{c}\left(t, t_{0}\right) \\
& \leq c_{2}\left\|\psi\left(t_{0}\right) x_{0}\right\|^{q} e_{c}\left(t_{1}, t_{0}\right) \\
& \leq M_{1}\left\|\psi\left(t_{0}\right) x_{0}\right\|^{q} e_{-\lambda}\left(t_{1}, t_{0}\right) e^{-\alpha c} e_{c}\left(t_{1}, t_{0}\right) \\
& <M_{1}\left\|\psi\left(t_{0}\right) x_{0}\right\|^{q} e_{-\lambda}\left(t_{1}, t_{0}\right), \quad t \in\left[t_{0}, t_{1}\right] \cap \mathbb{T}_{t_{0}} .
\end{aligned}
$$

Next, we show that

$$
V_{*}(t)<M_{1}\left\|\psi\left(t_{0}\right) x_{0}\right\|^{q} e_{-\lambda}\left(t_{2}, t_{0}\right), \quad t \in\left(t_{1}, t_{2}\right] \cap \mathbb{T}_{t_{0}} .
$$

From conditions (i)-(iv), (17) and Lemma 2, we have

$$
\begin{aligned}
V_{*}(t) & \leq V_{*}\left(t_{1}^{+}\right) e_{c}\left(t, t_{1}\right) \\
& \leq d_{1} V_{*}\left(t_{1}\right) e_{c}\left(t, t_{1}\right) \\
& \leq e_{-\lambda}\left(t_{2}, t_{1}\right) e^{-\lambda \alpha} M_{1}\left\|\psi\left(t_{0}\right) x_{0}\right\|^{q} e_{-\lambda}\left(t_{1}, t_{0}\right) e_{c}\left(t_{2}, t_{1}\right) \\
& \leq M_{1}\left\|\psi\left(t_{0}\right) x_{0}\right\|^{q} e_{-\lambda}\left(t_{2}, t_{0}\right), \quad t \in\left(t_{1}, t_{2}\right] \cap \mathbb{T}_{t_{0}} .
\end{aligned}
$$

Now we assume that (14) holds for $k=1,2, \ldots, m(m \in \mathbb{N})$; that is,

$$
\begin{aligned}
& V_{*}(t)<M_{1}\left\|\psi\left(t_{0}\right) x_{0}\right\|^{q} e_{-\lambda}\left(t_{k}, t_{0}\right), \\
& t \in\left(t_{k-1}, t_{k}\right] \cap \mathbb{T}_{t_{0}}, \quad k=1,2, \ldots, m .
\end{aligned}
$$


From conditions (iii) and (20), we have

$$
\begin{aligned}
V_{*}(t) \leq & V_{*}\left(t_{m}^{+}\right) e_{c}\left(t, t_{m}\right) \\
\leq & d_{m} V_{*}\left(t_{m}\right) e_{c}\left(t_{m+1}, t_{m}\right) \\
\leq & e_{-\lambda}\left(t_{m+1}, t_{m}\right) e^{-\lambda \alpha} M\left\|\psi\left(t_{0}\right) x_{0}\right\|^{q} e_{-\lambda}\left(t_{m}, t_{0}\right) \\
& \times e_{c}\left(t_{m+1}, t_{m}\right) \\
\leq & M_{1}\left\|\psi\left(t_{0}\right) x_{0}\right\|^{q} e_{-\lambda}\left(t_{m+1}, t_{0}\right), \quad t \in\left(t_{m}, t_{m+1}\right] \cap \mathbb{T}_{t_{0}} .
\end{aligned}
$$

Thus (14) holds for each $k=m+1$. Then it follows from mathematical induction that (14) holds for each $k \in \mathbb{N}$.

In view of conditions (i) and (14), we get

$$
\begin{aligned}
& \|\psi(t) x(t)\| \\
& \quad \leq M\left\|\psi\left(t_{0}\right) x_{0}\right\|^{\gamma}\left[e_{-\lambda}\left(t, t_{0}\right)\right]^{d}, \quad t \in\left(t_{k-1}, t_{k}\right] \cap \mathbb{T}_{t_{0}},
\end{aligned}
$$

where $M=\max \left\{1,\left(M_{1} / c_{1}\right)^{d}\right\}, \gamma=q / p$, and $d=1 / p$. Hence the trivial solution of system (2) is $\psi$-exponentially stable. This completes the proof.

Remark 9. We obtain the following results from Theorem 8.

(i) If we set $\psi(t)=1$ for each $t \in \mathbb{T}$ in Theorem 8 , then the zero solution of system (2) is exponentially stable.

(ii) If the conditions of Theorem 8 hold and $p=q$, then the zero solution of system (2) is globally $\psi$ exponentially stable.

Also, we can obtain the following result as a discrete version of Theorem 8 for $\mathbb{T}=\mathbb{Z}$.

Corollary 10. Assume that there exists a function $V: \mathbb{Z} \times$ $\mathbb{R}^{n} \rightarrow \mathbb{R}^{+}$and constants $p, q, c, c_{1}, c_{2}>0$ and $\alpha>1, c<\lambda<1$ such that the following conditions hold:

(i) $V(i, x)$ is locally Lipschizian in the second variable $x \in$ $\mathbb{R}^{n}$ and $V(i, 0)=0$ for each $i \in \mathbb{Z}$;

(ii) $c_{1}\|\psi(i) x\|^{p} \leq V(i, x) \leq c_{2}\|\psi(i) x\|^{q}$ for $(i, x) \in \mathbb{Z}_{i_{0}} \times \mathbb{R}^{n}$;

(iii) $V(i+1, x) \leq(1+c) V(i, x)$ for all $i \in\left(i_{k-1}, i_{k}\right] \cap \mathbb{Z}_{i_{0}}$;

(iv) $V\left(i_{k}^{+}, x\left(i_{k}\right)+I_{k}\left(x\left(i_{k}\right)\right)\right) \leq d_{k} V\left(i_{k}, x\left(i_{k}\right)\right)$, where each $d_{k}$ is a positive constant;

(v) $0<i_{k}-i_{k-1}<\alpha$ and $d_{k}<(1-\lambda)^{\left(i_{k+1}-i_{k}\right)} e^{-\alpha \lambda}$ for each $k \in \mathbb{N}$.

Then the zero solution of system (2) is $\psi$-exponentially stable.

We can obtain the following result which can be proved as in the similar manner of Theorem 8 .

Corollary 11. Assume that all conditions of Theorem 8 are satisfied with the condition (ii) replaced by $(i i)^{\prime}$ :

(ii) $^{\prime} V^{\Delta}(t, x) \leq 0$ for all $t \in\left(t_{k-1}, t_{k}\right] \cap \mathbb{T}_{t_{0}}, k \in \mathbb{N}$.

Then the zero solution of system (2) is also $\psi$-exponentially stable.
Remark 12. If we set $\psi(t)=1$ in the condition (i) of Corollary 11, then the zero solution of system (2) is also exponentially stable.

Next, we obtain the following result that the stability properties of dynamic systems can be preserved under certain impulsive perturbations. It is adapted from Theorem 1 in [13].

Theorem 13. Assume that there exist a function $V \in \nu_{0}$ and constants $p, q, c, c_{1}, c_{2}>0$ and $\alpha>0, \lambda>c$ with $-\lambda \in \mathscr{R}^{+}$ such that the following conditions hold:

(i) $c_{1}\|\psi(t) x\|^{p} \leq V(t, x) \leq c_{2}\|\psi(t) x\|^{q}$ for $(t, x) \in \mathbb{T}_{t_{0}} \times \mathbb{R}^{n}$;

(ii) $V^{\Delta}(t, x) \leq-c V(t, x)$ for all $t \in\left(t_{k-1}, t_{k}\right] \cap \mathbb{T}_{t_{0}}, k \in \mathbb{N}$;

(iii) $V\left(t_{k}^{+}, x\left(t_{k}\right)+I_{k}\left(x\left(t_{k}\right)\right)\right) \leq\left(1+d_{k}\right) V\left(t_{k}, x\left(t_{k}\right)\right)$, where each $d_{k}(k \in \mathbb{N})$ is a positive constant and $\sum_{k=1}^{\infty} d_{k}<$ $\infty$.

Then the zero solution of system (2) is $\psi$-exponentially stable.

Proof. Let $x(t)=x\left(t, t_{0}, x_{0}\right)$ be any solution of system (2) with $x\left(t_{0}\right)=x_{0}$, and $V_{*}(t)=V(t, x(t))$.

It follows from condition (ii) that

$$
\begin{aligned}
& {\left[V_{*}(t) e_{c}\left(t, t_{k}\right)\right]^{\Delta}} \\
& \quad=V_{*}^{\Delta}(t) e_{c}\left(\sigma(t), t_{k}\right)+V_{*}(t) e_{c}^{\Delta}\left(t, t_{k}\right) \\
& \quad \leq V_{*}^{\Delta}(t)(1+c \mu(t)) e_{c}\left(t, t_{k}\right)+c V_{*}(t) e_{c}\left(t, t_{k}\right) \\
& \quad \leq-c \mu(t) V_{*}(t) e_{c}\left(t, t_{k}\right) \\
& \quad \leq 0, \quad t \in\left(t_{k}, t_{k+1}\right] \cap \mathbb{T}_{t_{0}}, k \in \mathbb{N} .
\end{aligned}
$$

By integrating both sides of (23) from $t_{k}^{+}$to $t$ and condition (iii), we obtain

$$
V_{*}(t) \leq\left(1+d_{k}\right) V_{*}\left(t_{k}\right) e_{-c}\left(t, t_{k}\right), \quad t \in\left(t_{k}, t_{k+1}\right] \cap \mathbb{T}_{t_{0}} .
$$

From condition (ii), we have

$$
V_{*}(t) \leq V_{*}\left(t_{0}\right) e_{-c}\left(t, t_{0}\right), \quad t \in\left[t_{0}, t_{1}\right] \cap \mathbb{T}_{t_{0}} .
$$

In view of conditions (iii) and (25), we have

$$
\begin{aligned}
V_{*}(t) & \leq\left(1+d_{1}\right) V_{*}\left(t_{1}\right) e_{-c}\left(t, t_{1}\right), \\
& \leq\left(1+d_{1}\right) V_{*}\left(t_{0}\right) e_{-c}\left(t_{1}, t_{0}\right) e_{-c}\left(t, t_{1}\right) \\
& \leq\left(1+d_{1}\right) V_{*}\left(t_{0}\right) e_{-c}\left(t, t_{0}\right), \quad t \in\left(t_{1}, t_{2}\right] \cap \mathbb{T} t_{0} .
\end{aligned}
$$

It follows from mathematical induction that

$V_{*}(t)$

$$
\leq \prod_{i=1}^{k}\left(1+b_{i}\right) V_{*}\left(t_{0}\right) e_{-c}\left(t, t_{0}\right), \quad t \in\left(t_{k}, t_{k+1}\right] \cap \mathbb{T}_{t_{0}}, k \in \mathbb{N} .
$$


Thus we obtain

$$
\begin{aligned}
V_{*}(t) & \leq \prod_{i=1}^{\infty}\left(1+b_{i}\right) V_{*}\left(t_{0}\right) e_{-c}\left(t, t_{0}\right) \\
& \leq \exp \left(\sum_{i=1}^{\infty} b_{i}\right) c_{2}\left\|\psi\left(t_{0}\right) x_{0}\right\|^{q} e_{-c}\left(t, t_{0}\right) \\
& \leq M_{1} c_{2}\left\|\psi\left(t_{0}\right) x_{0}\right\|^{q} e_{-c}\left(t, t_{0}\right), \quad t \in \mathbb{T}_{t_{0}},
\end{aligned}
$$

where $M_{1}=\exp \left(\sum_{i=1}^{\infty} b_{i}\right)$.

In view of conditions (i) and (28), we have

$$
\|\psi(t) x(t)\| \leq M\left\|\psi\left(t_{0}\right) x_{0}\right\|^{\gamma} e_{-c}\left[\left(t, t_{0}\right)\right]^{d}, \quad t \in \mathbb{T}_{t_{0}},
$$

where $M=\left(c_{2} M_{1} / c_{1}\right)^{1 / p}, \gamma=q / p$, and $d=1 / p$. The proof is complete.

Remark 14. We obtain the following results in [13] from Theorem 13.

(i) If we set $\psi(t)=1$ for each $t \in \mathbb{T}$ in Theorem 13, then the zero solution of system (2) is exponentially stable.

(ii) If we set $\psi(t)=1$ and $p=q$ in condition (i) of Theorem 13, then the zero solution of system (2) is exponentially stable.

\section{Examples}

In this section we give two examples which illustrate our results from the previous section. Let $\mathbb{Z}_{+}=\{0,1,2, \ldots\}$.

Example 15 (see [24, Example 2]). We consider the impulsive dynamic equation on time scales

$$
\begin{gathered}
x^{\Delta}(t)=3 x, \quad t \neq t_{k}, t \in \mathbb{T}_{t_{0}} ; \\
x\left(t_{k}^{+}\right)=x\left(t_{k}\right)+\left(e^{-8}-1\right) x\left(t_{k}\right), \quad t=t_{k} ; \\
x\left(t_{0}\right)=x_{0},
\end{gathered}
$$

where $t_{k}=k \in \mathbb{T}$ for each $k \in \mathbb{N}$ and $x_{0} \in \mathbb{R}$.

Let $\psi(t)=1$ and $V(t, x)=x^{2}$, then it follows that

$$
\begin{aligned}
V^{\Delta}(t, x(t)) & =x^{\Delta}(t) x(\sigma(t))+x^{\Delta}(t) x(t) \\
& =x^{\Delta}(t)\left(2 x+\mu(t) x^{\Delta}(t)\right) \\
& =x^{2}(t)(6+9 \mu(t)), \quad t \in \mathbb{T}, \\
V\left(t_{k}^{+}, x\left(t_{k}\right)+I_{k}\left(x\left(t_{k}\right)\right)\right) & =\left[x\left(t_{k}\right)+\left(e^{-8}-1\right) x\left(t_{k}\right)\right]^{2} \\
& =e^{-16} x\left(t_{k}\right)^{2} \leq e^{-16} V\left(t_{k}, x\left(t_{k}\right)\right) .
\end{aligned}
$$

We consider two cases: $\mathbb{T}=\mathbb{R}$ and $\mathbb{T}=\left\{t=i / 10: i \in \mathbb{Z}_{+}\right\}$.
Case $1(\mathbb{T}=\mathbb{R})$. Letting $d_{k}=e^{-16}, c=6, \lambda=7, \alpha=1.1$, $p=q=1, c_{1}=1 / 2, c_{2}=1$, we note that all conditions of Theorem 8 are satisfied. Hence the zero solution of system (30) is $\psi$-exponentially stable by Theorem 8 .

Case $2\left(\mathbb{T}=\left\{t=i / 10: i \in \mathbb{Z}_{+}\right\}\right.$with $\left.\mu(i / 10)=1 / 10\right)$. Then system (30) rewrites

$$
\begin{gathered}
x^{\Delta}\left(\frac{i}{10}\right)=3 x\left(\frac{i}{10}\right), \quad t \neq t_{k}, t=\frac{i}{10} \in \mathbb{T} ; \\
x\left(k^{+}\right)=x(k)+\left(e^{-8}-1\right) x(k), \quad t=t_{k} ; \\
x(0)=x_{0},
\end{gathered}
$$

where $t_{k}=k \in \mathbb{N}$. Then we have

$$
\begin{aligned}
V^{\Delta}(t, x) & =V^{\Delta}\left(\frac{i}{10}, x\right) \\
& =x^{2}\left(6+9 \mu\left(\frac{i}{10}\right)\right) \\
& \leq 7 V(t, x), \quad t=\frac{i}{10} \in \mathbb{T} .
\end{aligned}
$$

Letting $d_{k}=e^{-16}, c=7, \lambda=7.1, \alpha=1.1, p=q=1$, $c_{1}=1 / 2, c_{2}=1$, it follows that all conditions of Theorem 8 are satisfied. Hence the zero solution of system (32) is also $\psi$-exponentially stable by Theorem 8 .

Remark 16. It follows from Example 15 that the zero solution of system (30) without impulses is unstable; however, after impulsive effect, the zero solution becomes $\psi$-exponentially stable. This implies that impulses may be used to exponentially stabilize dynamic equations on time scales.

We give the following example to illustrate Theorem 13.

Example 17 (see [13, Example]). Let $t_{0} \in \mathbb{T}$ and $x\left(t_{0}\right)=$ $(c, d) \in \mathbb{R}^{2}$. We consider the impulsive dynamic system on time scales

$$
\begin{gathered}
x_{1}^{\Delta}(t)=\frac{x_{2}(t)}{1+x_{1}^{2}(t)}-2 x_{1}(t), \quad t \neq t_{k}, t \in \mathbb{T}_{t_{0}} ; \\
x_{2}^{\Delta}(t)=\frac{x_{1}(t)}{1+x_{2}^{2}(t)}-2 x_{2}(t), \quad t \neq t_{k}, t \in \mathbb{T}_{t_{0}} ; \\
x_{1}\left(t_{k}^{+}\right)=\sqrt{1+\frac{1}{k^{2}}} x_{1}\left(t_{k}\right), \quad t=t_{k} ; \\
x_{2}\left(t_{k}^{+}\right)=\sqrt{1+\frac{1}{k^{2}}} x_{2}\left(t_{k}\right), \quad t=t_{k} ; \\
x\left(t_{0}\right)=\left(x_{1}\left(t_{0}\right), x_{2}\left(t_{0}\right)\right)=(c, d),
\end{gathered}
$$


where $t_{k}=k \in \mathbb{T}$ for each $k \in \mathbb{N}$. In case $\mathbb{T}=\left\{t=i / 2: i \in \mathbb{Z}_{+}\right\}$ with $\mu(i / 2)=i / 2$, then the system (34) rewrites

$$
\begin{gathered}
x_{1}^{\Delta}\left(\frac{i}{2}\right)=\frac{x_{2}(i / 2)}{1+x_{1}^{2}(i / 2)}-2 x_{1}\left(\frac{i}{2}\right), \quad t \neq t_{k}, t=\frac{i}{2} \in \mathbb{T} ; \\
x_{2}^{\Delta}\left(\frac{i}{2}\right)=\frac{x_{1}(i / 2)}{1+x_{2}^{2}(i / 2)}-2 x_{2}\left(\frac{i}{2}\right), \quad t \neq t_{k}, t=\frac{i}{2} \in \mathbb{T} ; \\
x_{1}\left(k^{+}\right)=\sqrt{1+\frac{1}{k^{2}}} x_{1}(k), \quad t=t_{k} ; \\
x_{2}\left(k^{+}\right)=\sqrt{1+\frac{1}{k^{2}}} x_{2}(k), \quad t=t_{k} ; \\
x(0)=\left(x_{1}(0), x_{2}(0)\right)=(c, d),
\end{gathered}
$$

where $t_{k}=k \in \mathbb{N}$. Letting $V(t, x(t))=x_{1}^{2}(t)+x_{2}^{2}(t)$ and $\|x(t)\|=x_{1}^{2}(t)+x_{2}^{2}(t)$ and employing similar manner in [13, Example], it follows that the zero solution of (34) is exponentially stable.

\section{Conflict of Interests}

The authors declare that there is no conflict of interests regarding the publication of this paper.

\section{Acknowledgments}

The first author was supported by Research Fund for the Doctoral Program of Harbin University of Commerce (no. 14RW06). The third author was supported by Basic Science Research Program through the National Research Foundation of Korea (NRF) funded by the Ministry of Education (NRF-2013R1A1A2007585). The authors are thankful to the anonymous referees for their valuable comments and corrections to improve this paper.

\section{References}

[1] D. D. Bainov and P. S. Simeonov, Systems with Impulse Effect: Stability, Theory and Applications, Ellis Horwood and John Wiley, New York, NY, USA, 1989.

[2] A. Halanay and D. Wexler, Teoria Calitativa a Sistemelor Cu Impulsuri, Editura Acadeiei Republicii Socialiste Romania, Bucharest, Romania, 1968.

[3] V. Lakshmikantham, D. D. Baĭnov, and P. S. Simeonov, Theory of Impulsive Differential Equations, World Scientific, Singapore, 1989.

[4] B. Aulbach and S. Hilger, "A unified approach to continuous and discrete dynamics," in Qualitative Theory of Differential Equations (Szeged, 1988), vol. 53 of Colloquia Mathematica Societatis János Bolyai, pp. 37-56, North Holland, Amsterdam, The Netherlands, 1990.

[5] B. Aulbach and S. Hilger, "Linear dynamic processes with inhomogeneous time scale," in Nonlinear Dynamics and Quantum Dynamical Systems (Gaussig, 1990), vol. 59 of Mathematical Research, pp. 9-20, Akademie, Berlin, Germany, 1990.
[6] M. Bohner and A. Peterson, Dynamic Equations on Time Scales, An Introduction with Applications, Birkhäuser, Boston, Mass, USA, 2001.

[7] V. Lakshmikantham, S. Sivasundaram, and B. Kaymakcalan, Dynamic Systems on Measure Chains, Kluwer Academic Publishers, Boston, Mass, USA, 1996.

[8] S. K. Choi, Y. Cui, and N. Koo, "Variationally stable dynamic systems on time scales," Advances in Difference Equations, vol. 2012, article 129, 2012.

[9] Y. Chen and R. Tian, "Exponential stability of impulsive discrete systems with multiple delays," Journal of Networks, vol. 8, no. 11, pp. 2564-2571, 2013.

[10] B. Gupta and S. K. Srivastava, " $\psi$-exponential stability of non-linear impulsive differential equaions," World Academy of Science-Engineering and Technology, vol. 44, pp. 347-350, 2010.

[11] Q. Wang and X. Liu, "Impulsive stabilization of delay differential systems via the Lyapunov-Razumikhin method," Applied Mathematics Letters, vol. 20, no. 8, pp. 839-845, 2007.

[12] V. F. Hatipoğlu, D. Uçar, and Z. F. Koçak, " $\psi$-Exponential stability of nonlinear impulsive dynamic equations on time scales," Abstract and Applied Analysis, vol. 2013, Article ID 103894, 5 pages, 2013.

[13] H. Liu and X. Fu, "Exponential stability of impulsive dynamic systems on time scales," Science Technology and Engineering, pp. 685-689, 2011.

[14] V. Lupulescu and A. Zada, "Linear impulsive dynamic systems on time scales," Electronic Journal of Qualitative Theory of Differential Equations, vol. 2010, no. 11, pp. 1-30, 2010.

[15] Y. Ma and J. Sun, "Stability criteria for impulsive systems on time scales," Journal of Computational and Applied Mathematics, vol. 213, no. 2, pp. 400-407, 2008.

[16] I. B. Yasar and A. Tuna, " $\psi$-uniformly stability for time varying linear dynamic systems on time scales," International Mathematical Forum, vol. 2, pp. 963-972, 2007.

[17] X. Liu, "Impulsive stabilization of nonlinear systems," IMA Journal of Mathematical Control and Information, vol. 10, no. 1, pp. 11-19, 1993.

[18] M. Benchohra, J. Henderson, S. K. Ntouyas, and A. Ouahab, "On first order impulsive dynamic equations on time scales," Journal of Difference Equations and Applications, vol. 10, no. 6, pp. 541-548, 2004.

[19] A. Liu, Qualitative properties and stability analysis for dynamic equations on time scales [Ph.D. thesis], Sun Yat-Sen University, 2005.

[20] O. Akinyele, "On partial stability and boundedness of degree $k$," Atti della Accademia Nazionale dei Lincei: Rendiconti: Classe di Scienze Fisiche, Matematiche e Naturali: Serie VIII, vol. 65, no. 6, pp. 259-264, 1978.

[21] T. Yoshizawa, Stability Theory by Liapunov's Second Method, The Mathematical Society of Japan, 1966.

[22] C. Pötzsche, "Chain rule and invariance principle on measure chains," Journal of Computational and Applied Mathematics, vol. 141, no. 1-2, pp. 249-254, 2002.

[23] X. Liu and Q. Wang, "The method of Lyapunov functionals and exponential stability of impulsive systems with time delay," Nonlinear Analysis: Theory, Methods \& Applications, vol. 66, no. 7, pp. 1465-1484, 2007.

[24] W. Z. Feng and Y. S. Chen, "Weak exponential asymptotic stability of an impulsive differential system," Applied Mathematics: A Journal of Chinese Universities: Series A, vol. 17, no. 1, pp. 1-6, 2002. 


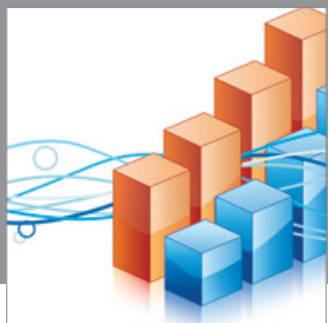

Advances in

Operations Research

mansans

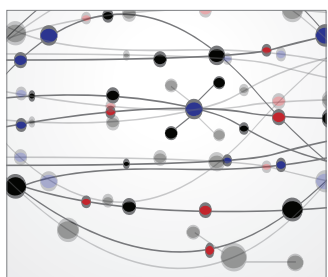

The Scientific World Journal
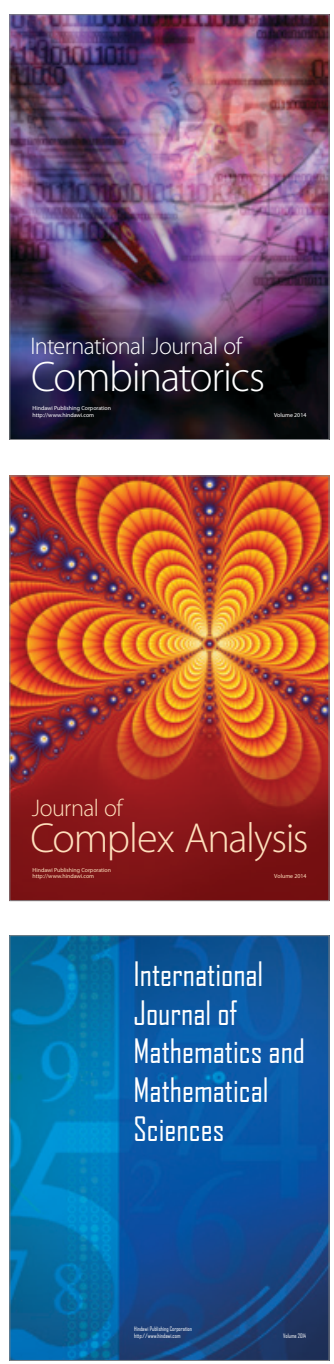
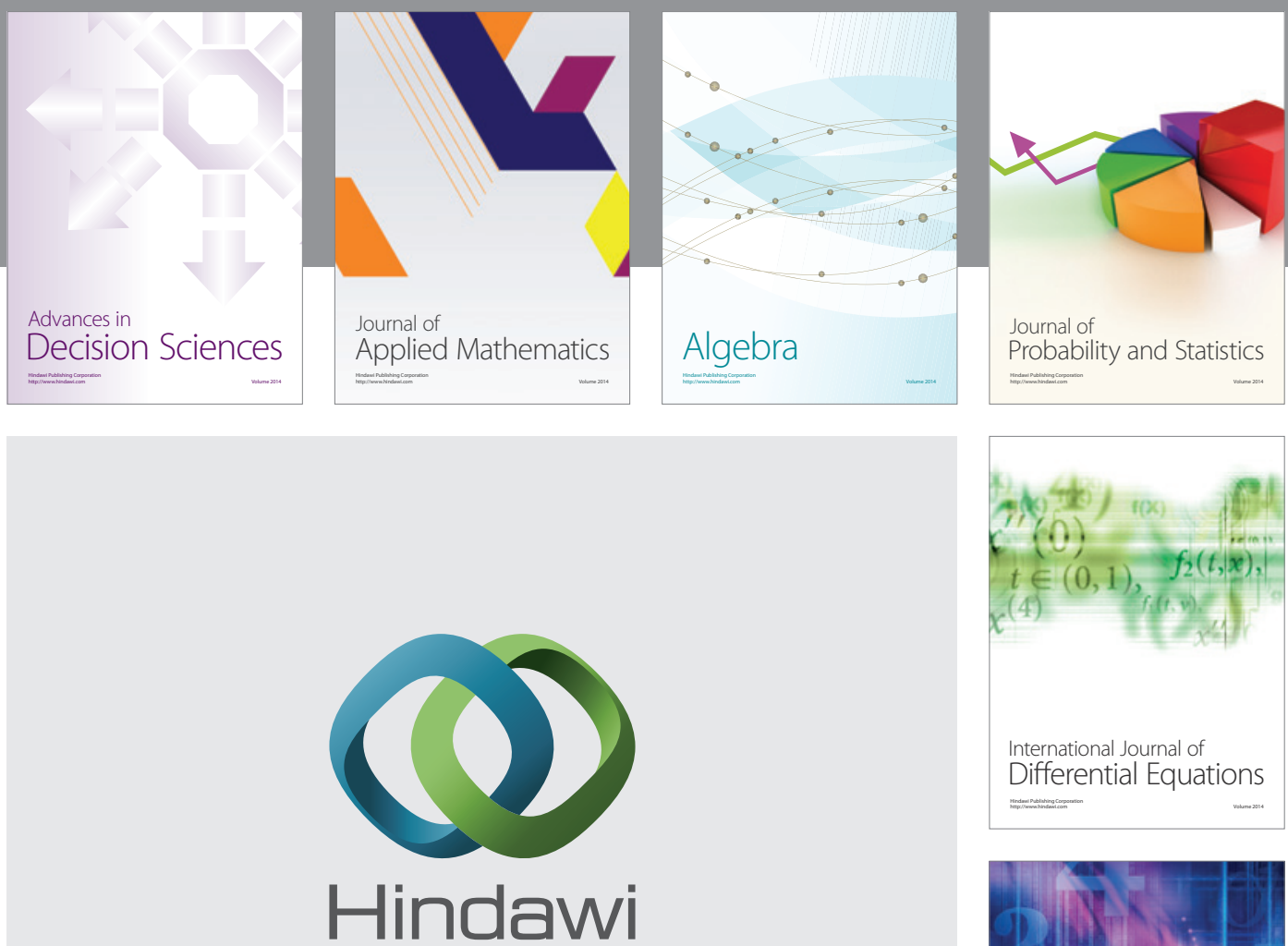

Submit your manuscripts at http://www.hindawi.com
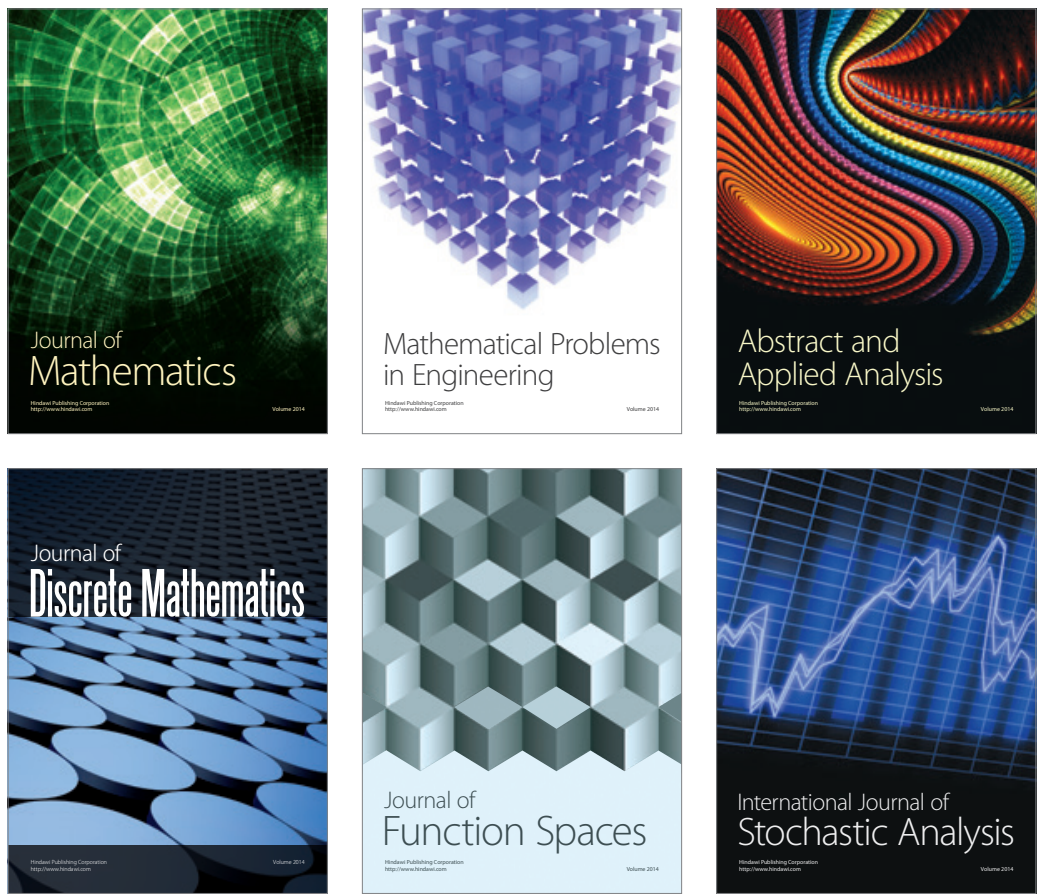

Journal of

Function Spaces

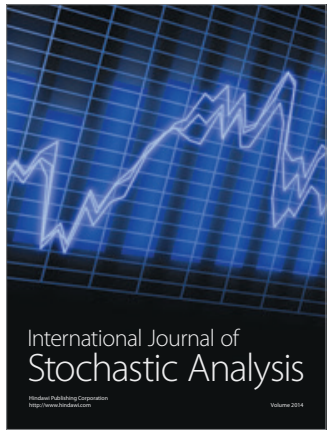

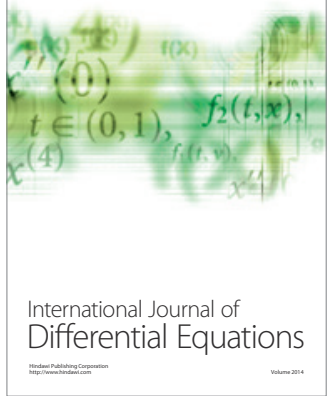
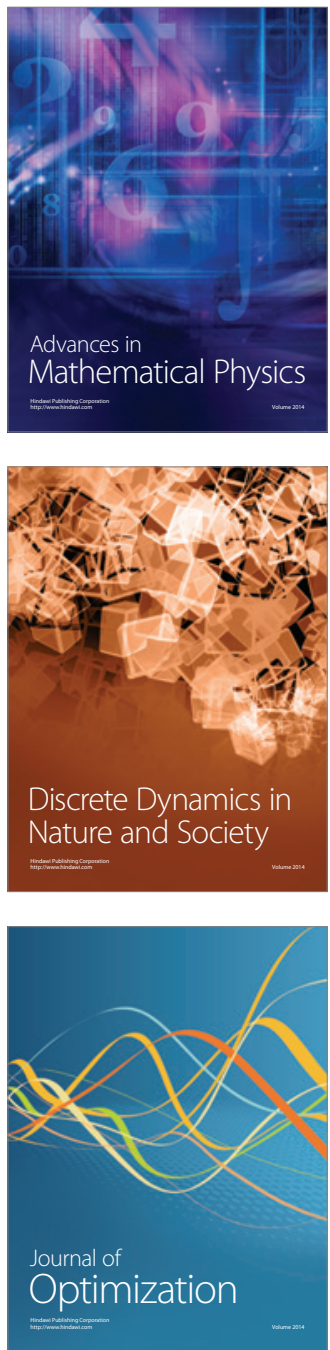